surveillance data in Canadian hospitals based on problematic data and methods used.

Acknowledgments.

Financial support. No financial support was provided relevant to this article.

Conflicts of interest. All authors report no conflicts of interest relevant to this article.

\section{References}

1. Ramirez Mendoza JY, Daneman N, Elias MN, Amuah JE, Buth K, Couris $\mathrm{CM}$ et al. A comparison of administrative data versus surveillance data for hospital-associated methicillin-resistant Staphylococcus aureus infections in Canadian hospital. Infect Control Hosp Epidemiol 2017;38: 436-443.

2. Watson FP, Petrie A. Method agreement analysis: a review of correct methodology. Theriogenology 2010;73:1167-1179.

3. Tang KL, Lucyk K, Quan H. Coder perspectives on physician-related barriers to producing high-quality administrative data: a qualitative study. CMAJ 2017;5:E617-E621.

4. Nicholls SG, Langan SM, Benchimol EI. Routinely collected data: the importance of high-quality diagnostic coding to research. CMAJ 2017 August 21;189:E1054-E1055.

5. Lin's concordance correlation coefficient. Marta Garcia-Granero SPSS Macros. https://gjyp.nl/marta/. Published 2010. Accessed April 2017.

\title{
Going back to prospectively collected results with a probiotic for primary prevention of Clostridium difficile infection at a tertiary-care medical center
}

\author{
Noam Ship PhD and Serge Carrière MD, FRCP \\ Research and Development, Bio-K Plus International, Laval, Quebec, Canada
}

To the Editor-Implementing a facility-level Clostridium difficile infection (CDI) prevention protocol is a challenging endeavor, and Trick et $\mathrm{al}^{1}$ should be commended for their early successes at a large institution. As the manufacturers of the probiotic comprising L. acidophilus CL1285, L. casei LBC80R, and L. rhamnosus CLR2 (Bio-K+), we contributed our products to 9,072 patients for this study at no cost to the investigators as well as monetary support for a research assistant to collect data. We watched the evolution of this quality improvement study in anticipation, receiving regular updates on recruitment and product consumption, though we had no role in the collection or interpretation of the data.

Several issues emerged that made the investigators "unable to electronically extract patient-level antibiotic and probiotic receipt data." In the absence of a mechanism to review the full dataset of thousands of eligible patients, the authors undertook a case-control study to examine 68 cases and 68 matched controls in detail using a retrospective chart review. One conclusion from this study was that after adjusting for severity of illness, and temporal and spatial proximity, there was "no protective effect from the probiotic." It is our opinion that the case-control study was rigorous in considering the available data. Many of the known risk factors were controlled, but the principal modifiable risk factor for CDI-antibiotics-was mostly overlooked. Tartof et $\mathrm{al}^{2}$ elegantly showed a 2 -fold increase in the risk of CDI with each additional antibiotic administered. Among 401,234 adults admitted to Kaiser Permanente Southern California hospitals, $0.5 \%$ tested CDI-positive when taking 1 antibiotic,

Author for correspondence: Dr Serge Carrière, Bio-K Plus International, 495 Boul. Armand-Frappier, Laval, Québec, H7V 4B3, Canada. Email: scarriere@biokplus.com.

Cite this article: Ship N and Carrière S (2018). Going back to prospectively collected results with a probiotic for primary prevention of Clostridium difficile infection at a tertiary-care medical center. Infection Control \& Hospital Epidemiology 2018, 39, 1392-1393. doi: 10.1017/ice.2018.205
$1.0 \%$ when taking 2 antibiotics, and $2.3 \%$ when taking 3 or more antibiotics.

It may be, for example, that the composition of a patient's intestinal microbiota prior to antibiotic exposure is another relevant predictor of susceptibility to $C$. difficile overgrowth and infection. A detailed metagenomics analysis by Raymond et $\mathrm{al}^{3}$ of stool samples from healthy volunteers taking an antibiotic uncovered consistent impairments to the diversity and functioning of the gut microbiota and enrichment of resistance genes. In addition, the initial microbiome composition among certain volunteers predicted an overgrowth of known pathogens. Applied to the hospital setting, it could be that hospitalized patients who develop a CDI are inherently more susceptible to the effect of antibiotics on their microbiome. Scientific questions like these are beyond the technical limitations of the case-control design.

The case-control study did not detect a protective effect, but results from the same cohort presented in 2015 indicate a reduced risk of CDI in patients treated with this probiotic. ${ }^{4}$ A risk ratio of $0.6(95 \% \mathrm{CI}, 0.4-0.9 ; P=.01)$ was calculated, representing a significant protective effect from $C$. difficile infection for the probiotic recipients (Fig. 1). The inputs for this equation are based on the principal data set collected prospectively within the design of the study, and as such, they do not rely on detailed electronic patient records. Thus, these prospectively collected data suggest that the intervention was having the intended effect.

Although Trick et al describe challenges in implementing the protocol, in following patients, and in extracting patientlevel data, this quality improvement study provides invaluable information on the real-world practical application of probiotics in the fight against $C$. difficile infection. As noted in a public release by the Society for Healthcare Epidemiology of America (SHEA), it is critically important to test emerging interventions in routine practice and to learn from those 


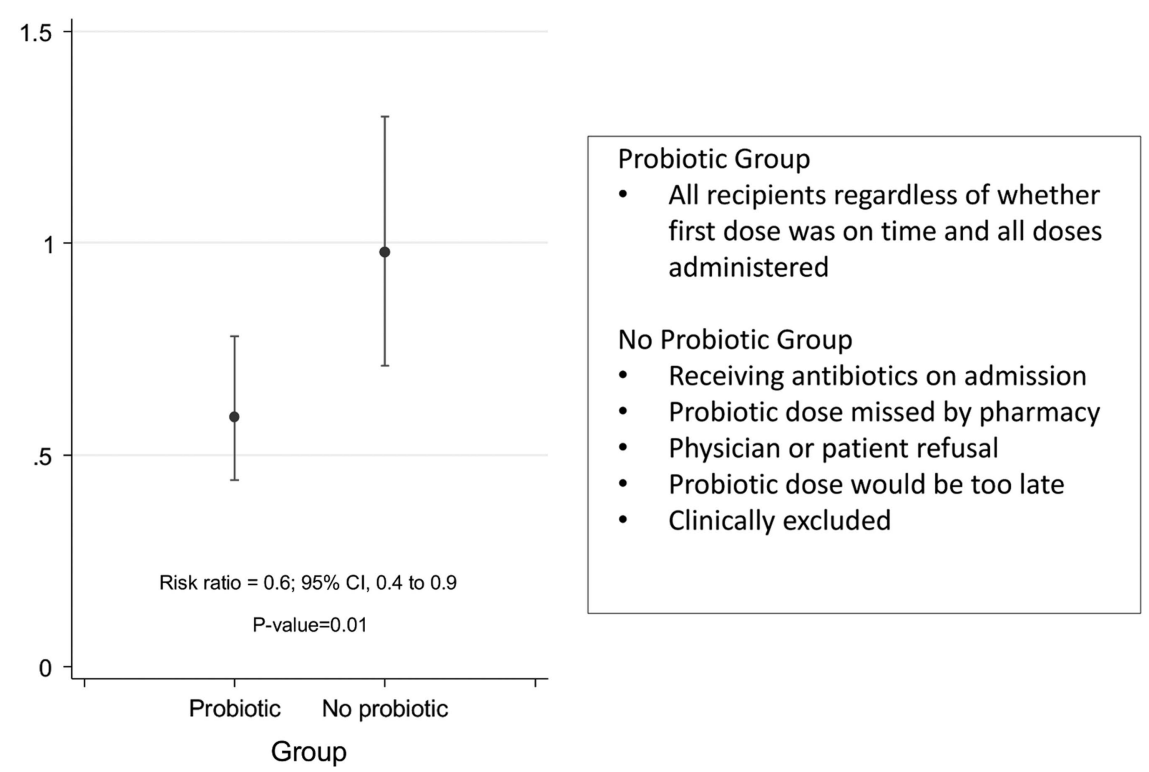

Fig. 1. Comparison of rates of Clostridium difficile infection (CDI) between probiotic recipients and antibiotic recipients who did not receive a probiotic. [Reproduction from a poster presented at ID Week 2015. $]^{4,5}$

experiences. ${ }^{6}$ Considering the relative safety and moderate cost of probiotics, practical experiences regarding probiotics implemented for primary CDI prevention are scarce. McFarland et $\mathrm{al}^{7}$ recently reviewed the literature evaluating this probiotic $($ Bio- $\mathrm{K}+)$ and idenitified 7 accounts of its implementation at the facility-level for CDI prevention and a few instances with other probiotic formulations. Moving forward, hospitals can learn from the experiences of Trick et al to better address their patients' vulnerability to CDI with another line of defense.

Acknowledgments. N.S. and S.C. had no role in the collection or interpretation of data published by Trick et al (reference 1).

Financial support. No financial support was provided relevant to this article.

Conflicts of interest. N.S. and S.C. are employees of Bio-K Plus International, the company that partially sponsored the study in question and commercializes the study product.

\section{References}

1. Trick WE, Sokalski SJ, Johnson S, et al. Effectiveness of probiotic for primary prevention of Clostridium difficile infection: a single-center before- and-after quality improvement intervention at a tertiary-care medical center. Infect Control Hosp Epidemiol 2018;39:765-770.

2. Tartof SY, Rieg GK, Wei R, Tseng HF, Jacobsen SJ, Yu KC. A comprehensive assessment across the healthcare continuum: risk of hospital-associated Clostridium difficile infection due to outpatient and inpatient antibiotic exposure. Infect Control Hosp Epidemiol 2015;36: 1409-1416.

3. Raymond F, Ouameur AA, Deraspe M, et al. The initial state of the human gut microbiome determines its reshaping by antibiotics. ISME J 2016;10:707-720.

4. Trick W, Sokalski S, Levato J, et al. Hospital-wide evaluation of probiotic administration as primary prevention of Clostridium difficile infection (CDI) in a tertiary care hospital. In Open Forum Infect Dis 2015;2,S1:971.

5. Trick W, Sokalski S, Levato J, et al. Hospital-wide evaluation of probiotic administration as primary prevention of Clostridium difficile infection (CDI) in a tertiary care hospital. Infectious Diseases Society of America, ID Week 2015 website. https://idsa.confex.com/idsa/2015/webprogram/Handout/ id3319/POSTER137_971.pdf. Published 2015. Accessed June 13, 2018.

6. Probiotics useful in the fight against $C$. difficile. Society for Healthcare Epidemiology of America website. https://www.shea-online.org/index. php/journal-news/press-room/press-release-archives/588-probiotics-useful-in-the-fight-against-c-difficile. Published 2018. Accessed June 13, 2018.

7. McFarland LV, Ship N, Auclair J, Millette M. Primary prevention of Clostridium difficile infections with a specific probiotic combining Lactobacillus acidophilus, L. casei, and L. rhamnosus strains: assessing the evidence. J Hosp Infect 2018;99(4):443-452. 